\title{
Multiple equilibria in symmetric strategies for simultaneous auctions in next-generation bandwidth markets
}

\author{
Fernando Beltrán and Matthias Roggendorf \\ University of Auckland Business School \\ 7 Symonds Street \\ Auckland, New Zealand \\ f.beltran|m.roggendorf@auckland.ac.nz
}

\begin{abstract}
We investigate equilibrium properties of a bidding strategy in a situation in which bidders attempt to purchase a given amount of a divisible resource by bidding on multiple auctions. Each seller uses the Progressive Second-Price auction. A domain of application is the next-generation of wireless networks, in which multiple, competing network providers sell bandwidth to multiple users able to aggregate their purchased bandwidth shares. Every bidder needs to coordinate his bids to maximise the utility derived from all auction. In order to deal with aggregation and coordination of bids, we introduce the notion of an aggregate market, which is an artificial construct that each bidder uses to distribute his demand among the auctions. Furthermore, the aggregate market helps to understand convergence of the bidding process occurring at each individual auction. We show that by using an incentive-compatible, efficient mechanism at each single auction bidders have incentives to truthfully reveal their demand to the aggregate market.
\end{abstract}

\section{INTRODUCTION}

When bidders' values are private, the Vickrey-Clarke-Groves (VCG) mechanism is a direct, incentive-compatible and efficient mechanism, implying that VCG calls for truthful revelation of bidder's values [2]. When the object being auctioned is divisible, the VCG mechanism requires a bidders to reveal their entire valuation function. Closely related to VCG for divisible goods is the Progressive Second-Price (PSP) auction, in which the the messages are reduced to a price-quantity combination [7]. The resulting game becomes iterative with all bidders reacting to the price-quantity combinations submitted by the opponents. In addition, the strong properties of VCG are relaxed in terms of the equilibrium concept.

In its original introduction PSP is a mechanism to allocate bandwidth among competing users of a communication

Permission to make digital or hard copies of all or part of this work for personal or classroom use is granted without fee provided that copies are not made or distributed for profit or commercial advantage and that copies bear this notice and the full citation on the first page. To copy otherwise, to republish, to post on servers or to redistribute to lists, requires prior specific permission and/or a fee.

GameComm '07, October 22, 2007, Nantes, France

Copyright 2007 ICST 978-963-9799-00-4. network. On a broader view, PSP is a decentralized and distributed market mechanism for resource sharing in networks. The use of a market mechanism stems from the need to acknowledge that different users may have different valuations for the resource. It is also used as a key part to the provision of Quality of Service (QoS). Selfish users attempting to gain resources, such as bandwidth, from a network provider, will contribute to the state of congestion above which QoS may be compromised. PSP is a mechanism by which prices constitute a dynamic response to (unpredictable) demand, while keeping a trade-off between engineering efficiency and economic efficiency.

We are interested in extending the use of PSP to a context in which users with elastic demand compete to obtaining a sought amount of a resource, which they may procure in shares bought from multiple vendors. We introduce a model of bidding on multiple auctions, each of which runs a PSP auction. Such a situation is likely to arise in the next generation of wireless network (NGWN), whose vision is described as a truly seamless, multi-technology, multi-provider network, serving mobile and fixed users with a variety of adaptive multi-media services [1]. The adaptation of applications and services is the central element and is equated to the need that each element best uses its resources. The latter is posing many challenges to network operators such as the dynamic allocation of scarce network resources in such a heterogeneous system. The next generation of wireless access networks will introduce the possibility of having simultaneous access to possibly competing networks, allowing users to seamlessly switch between network providers, even during ongoing service sessions. The subject of this paper can be translated into NGWN as the need to model the access to competing wireless providers all of which use an auction for resource allocation. A user may then aggregate shares of the resource obtained from several providers.

In this paper we investigate equilibrium properties of a bidding strategy, first defined in [5], in a situation in which bidders attempt to purchase a given amount of a divisible resource by bidding on multiple auctions. A bidder may aggregate shares of the resource obtained from some of the auctions, and needs to coordinate his bids to optimise the overall utility derived from each auction. In order to deal with aggregation and coordination of bids, we introduce the notion of an aggregated market, which can be seen as an artificial construct by each bidder to coordinate his bidding behaviour. Furthermore, this construct allows us to understand convergence of the bidding process occurring at each 
individual auction. Auction rules at each single auction provide incentives to bidders for truthful revelation to the aggregated market

The rest of the paper is organised as follows: Section 2 briefly considers the main questions that arise from the proposed model of resource allocation. Section 3 revisits the Progressive Second-Price auction. Section 4 presents the BalancedBid strategy, discussing the aggregated market, an artificial construct by each bidder that allows to solve the bid-splitting problem that bidders face; in there we also address the equilibrium properties of the strategy and efficiency of the solution. Section 4 draws the main conclusions of our work and exposes some avenues for future research.

\section{ON BIDDING ACROSS MULTIPLE MAR- KETS}

The literature on bidding strategies for multiple auctions of divisible goods is limited. The authors in [9] develop a detailed analysis of the problem of finding the optimal bidding strategy when a bidder is faced with multiple auctions occurring simultaneously. The paper aims at modelling the coordination of bidding activities across multiple auction markets where all sellers either use first-price or second-price sealed-bid auctions.

For simplicity, the authors consider the case of two auctions and an unspecified number of bidders. They assume that each of the items is sold through an independently run first-price sealed-bid auction. An optimal bidding function is derived using an analysis based on a game against nature. In such a game, the bids of rival bidders are part of the uncertain environment and, therefore, for a given player they hypothesise the existence of two probability distributions, $G 1$ and $G 2$ which describe the probability of player winning auction 1 and 2, respectively. An additional assumption is the introduction of the extra utility factor generated for a bidder by acquiring both items. When such extra utility factor is zero, the auctions seem independent to the bidder, that is, the bidder would just go to each auction to try to win the item without regard to any complementarity that may arise from winning both items. In this particular case, their results for the optimal bidding strategy coincide with the optimal bidding strategy derived for a bidder in a firstprice auction when a Bayesian-Nash equilibrium approach is used. In both approaches, both bidders' values and the functions $G 1$ and $G 2$ are assumed to be uniform.

When the authors in [9] refer to the second-price sealedbid auction, they also find that the optimal bidding coincides with the optimal bidding for the second-price auction in the Bayesian-Nash approach, under the same assumptions for the complementarities between items, and the value and $G 1$ and $G 2$ functions. They also present a simple case of two bidders and two objects in which the optimisation has a simple, closed-form solution.

The objective of each bidder participating in the market is to maximise the individual utility derived from all auctions. In order to achieve this goal each bidder seeks to find his best bidding strategy for submitting bids in some or all auctions. When faced with the possibility of bidding at more than one auction the bidder is first confronted with selecting the auctions he wants to actively participate in, and then to select the optimal bid for each auction, or, altogether the optimal combination of bids for the auctions chosen.
Assuming that two competitive sellers use the same auction type, our research focuses on the following questions:

- If a user needs to acquire a given amount of resource and is able to satisfy his need by aggregating from more than one source, how should he bid at each of the two auctions?

- What are the effects of bid-splitting on a bidder's expected utility?

While for a single market with one provider the PSP auction is well defined some questions remain unanswered when more than one provider offers resources in a multi-auction market:

- How can a user express his true valuation when giving the choice of more than one seller?

- How can overall efficiency be assured when running PSP at each seller?

In PSP users adjust their bids once they receive information on the bid profile of the opponent bidders. Bidders are therefore allowed to update their bids, deciding whether they bid again whenever they find the new bid improves on their current utility. Although auctions are considered simultaneous (because they are active for all users at the same time and no bidder has final information of an auction before entering another), the fact that PSP is the focus of our analysis introduces an element of sequencing as PSP allows for bid recalculation by every bidder once they are informed about the bid profile of their opponents.

\section{THE PROGRESSIVE SECOND-PRICE AUC- TION}

Let us suppose that the seller has $Q$ units to be sold. The PSP auction proceeds with a bidder $i \in\{1, . ., I\}$ submitting a bid $s_{i}$ consisting of a pair $s_{i}=\left(p_{i}, q_{i}\right)$ of values: the amount $q_{i}$ of resource requested and the price $p_{i}$ for one unit of the resource. The PSP allocation mechanism sorts the incoming bids according to the unit price indicated by the bidder and allocates resources to all bidders up to the total quantity $Q$. The auctioneer allocates a share $a_{i}$ of the resource to player $i$ at the $\operatorname{cost} c_{i}$. The allocation rule assigns player $i$ an amount $a_{i}$ equal to the minimum value between his bid, $q_{i}$, and the remaining amount after all those bids, $q_{k}$, whose prices beat $i^{\prime} s$ bid $\left(p_{k} \geq p_{i}\right)$ are subtracted from the total amount $Q$ to be allocated. In other words, the allocation rule is $[8]^{1}$ :

$$
a_{i}(s)=q_{i} \wedge \frac{q_{i}}{\sum_{k ; p_{k}=p_{i}} q_{k}} Q\left(p_{i} ; s_{-i}\right)
$$

with $Q\left(y, s_{-i}\right)=\left[Q-\sum_{p_{k} \geq p_{i}, k \neq i} q_{k}\right]^{+}$and $s$ representing the set of bids by $i$, denoted as $s_{i}$ and by the rest of the players, denoted as $s_{-i}$.

The payment of any agent $i$ follows the "exclusion compensation" principle to cover the social opportunity costs of participating in the auction. Therefore, the total payment of

${ }^{1}$ Note that the allocation rule presented here is a slightly modified version of Semret's work, which appeared in [8] to correct some formal problems in the case of bid ties. 
each agent is the sum of the willingness-to-pay of all players excluded by $i$ 's presence.

$$
c_{i}(s)=\sum_{j \neq i} p_{j}\left[a_{j}\left(s_{-i}\right)-a_{j}\left(s_{i} ; s_{-i}\right)\right]
$$

The allocation rule applied with all players has been proved to lead to an equilibrium, which meets the first design objective. The algorithm has a bidder selecting a bid as a point (quantity, price), on his demand function; by relaxing the stringent equilibrium concept - with the introduction of the $\epsilon$-Nash equilibrium - a bidder is forced to submit a new bid that improves his utility by at least an amount $\epsilon$. The latter means that in order to guarantee the convergence of the algorithm a bidding fee $\epsilon$ has been introduced to let bidders change their bids only when the gain in net benefit is large enough.

With incentive compatibility in PSP auctions, it is the best strategy for all bidders to truthfully report their valuation. Therefore, the bidding behaviour can be modelled by using the individual demand function of each bidder. After receiving signals about the last auction results, a player $i$ may decide to change his current bid to increase his chances of winning a share of network capacity. By evaluating the opponents' bid profile he can decide on an optimal quantity he should bid in the next round. His demand function then defines the marginal unit price he can bid. Having found the new quantity and unit price $\left(p_{i}, q_{i}\right)$ the bidder calculates the utility of this new bid and compares it to the utility of the outcome obtained in the last auction round $\left(a_{i}, c_{i}\right)$, with $a_{i}$ being the assigned quantity and $c_{i}$ the total cost. If the utility of the truthful reply exceeds the auction outcome by more than /epsilon, the bidder decides to submit this new bid. It needs to be stressed that the concept only applies for shortsighted bidders, which do not anticipate the strategy of their opponents.

\subsection{The BalancedBid bidding strategy for a multi- auction market}

In this section we study the formal equilibrium characteristics of multiple independently managed auctions when bidders are allowed to split their bids in order to aggregate shares of the resource from several auctions. Assuming PSP is used by each auctioneer, we are concerned with the convergence to equilibrium at each auction when every bidder uses a bidding strategy known as BalancedBid [5].

Before presenting the bidding strategy in detail and discussing its main properties some notation is introduced in the following.

Let us assume the set of bidders is $1, . ., I$, the set of auctions is $1, \ldots, J$ and $Q^{(j)}$ units are being sold at auction $j$. Bidder $i$ 's bid on auction $j$ is $s_{i}^{(j)}=\left(q_{i}^{(j)}, p_{i}^{(j)}\right) \in S_{i}^{(j)}=$ $\left[0, Q^{(j)}\right] \times[0, \bar{P}]$ with $\bar{P}$ an upper bound on the unit price. Let us also define $S_{i}=\prod_{j} S_{i}^{(j)}$ as the set of all possible bids of bidder $i$ at the auctions. A composite or split bid $s_{i}=\left(s_{i}^{(1)}, . ., s_{i}^{(J)}\right)$ from bidder $i$ is a point in $S_{i}$. We assume that bidder i submits a bid to all or some of the auctions at the same time.

To model a reserve price in each auction an additional player $i=0$ is introduced, which bids with a fixed valuation $\theta_{0}=p_{0} Q^{(j)}$. This bidder is present in each auction and $p_{0}$ determines the minimum bid needed by other bidders to receive resources.
In the same fashion as $s_{i}^{(j)}$, bidder $i$ 's opponent bid profile at auction $j$ can be written as

$$
s_{-i}^{(j)}=\left[s_{0}^{(j)}, \ldots, s_{i-1}^{(j)}, s_{i+1}^{(j)}, . ., s_{I}^{(j)}\right] \in S_{-i}^{(j)}=\prod_{n \neq i} S_{n}^{(j)},
$$

where $S_{-i}^{(j)}$ is a set of all possible opponent bid profiles, consisting of the bids submitted by all bidders $n \neq i$ at the last (most recent) auction round. We summarise all opponent bid profiles from all auctions into a matrix $s_{-i}$, with

$$
\begin{aligned}
& s_{-i}=\left(\begin{array}{c}
s_{-i}^{(1)} \\
. \\
s_{-i}^{(J)}
\end{array}\right)=\left(\begin{array}{cccccc}
s_{0}^{(1)}, & . ., & s_{i-1}^{(1)}, & s_{i+1}^{(1)}, & . ., & s_{I}^{(1)} \\
. . & & & & \\
s_{0}^{(J)}, & . ., & s_{i-1}^{(J)}, & s_{i+1}^{(J)}, & . ., & s_{I}^{(J)}
\end{array}\right)
\end{aligned}
$$

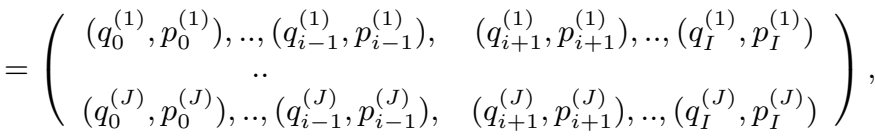

and define $S_{-i}=\prod_{j=\{1, \ldots, J\}} S_{-i}^{(j)}$.

As in the one-auctioneer case a player $i$ has a valuation function $\theta_{i}$ for the resource. If, at any given time, $i$ has been allocated shares $a_{i}^{(1)}, . ., a_{i}^{(J)}$ from the $J$ auctions and is supposed to pay $c_{i}^{(1)}, . ., c_{i}^{(J)}$ to every individual auctioneer $j$, his utility is given by $u_{i}\left(a_{i}, c_{i}\right)=\theta_{i}\left(\sum_{j=1}^{J} a_{i}^{(j)}\right)-\sum_{j=1}^{J} c_{i}^{(j)}$, that is, the valuation for the sum of all resources obtained from each auction minus the costs from each auction.

We also need to make some assumptions about a bidder's utility function.

Assumption 3.1. As in [7], the following assumptions on $\theta_{i}, \forall i \in I$, hold:

- $\theta_{i}(0)=0$,

- $\theta_{i}$ is differentiable,

- $\theta_{i}^{\prime} \geq 0$, non-increasing and continuous,

- $\exists \gamma_{i}>0, \forall z \geq 0, \theta_{i}^{\prime}(z)>0 \Rightarrow \forall \eta<z, \theta_{i}^{\prime}(z) \leq \theta_{i}^{\prime}(\eta)-$ $\gamma_{i}(z-\eta)$.

Assumption 3.2. $\exists \kappa>0, \forall i \in I$,

- $\forall z, z^{\prime}, z>z^{\prime} \geq 0, \theta_{i}^{\prime}(z)-\theta^{\prime}\left(z^{\prime}\right)>-\kappa\left(z-z^{\prime}\right)$,

- $\theta_{i}^{\prime}<\infty$,

With this notation we can define the game played by a bidder with access to multiple PSP auctions. ${ }^{2}$

DEFINITION 3.1. (A game of multiple PSP auctions) The normal-form representation $G$ of the auction game, which is played by the bidders with access to multiple PSP auctions is given by:

$$
G=\left(S_{1}, . ., S_{I}, u_{1}, . ., u_{I}\right),
$$

with $S_{i}$ being the strategy space of player $i$ defined as $S_{i}=$ $\prod_{j} S_{i}^{(j)}$, and $u_{i}$ being the utility of player $i$.

We are interested in finding a Nash equilibrium of the game given in Definition 3.1 under complete information.

${ }^{2}$ All allocation rules $A^{(j)}$ for each individual auction $j$ are identical to the original PSP auction as defined in [7]. 
DeFinition 3.2. (Nash equilibrium)

The strategies $\left(s_{1}^{*}, . ., s_{I}^{*}\right)$ are a Nash equilibrium if, for each player $i, s_{i}^{*}$ is player $i$ 's best response to the strategies specified for the $I-1$ other players $s_{-i}^{*}$ :

$$
u_{i}\left(s_{i}^{*} ; s_{-i}^{*}\right) \geq u_{i}\left(s_{i}^{\prime} ; s_{-i}^{*}\right),
$$

for every feasible strategy $s_{i} \in S_{i}$; that is, $s_{i}^{*}$ solves:

$$
\max _{s_{i} \in S_{i}} u_{i}\left(s_{i}^{*} ; s_{-i}^{*}\right) \text {. }
$$

In an iterative ${ }^{3}$ game, where players recompute their best response based on a modified opponent bid profile, the bid profile can either converge to a Nash equilibrium or not converge at all [7]. In contrast to the single-auction case the problem is now to identify the strategy of player $i$, consisting of $J$ bids to be submitted to each auction $j$.

To let the auctions converge in finite time we use Semret's notion of an $\epsilon$-Nash equilibrium [7], which allows a bidder to stop updating a bid profile once the difference in utility provided by the current composite bid and the next one is less than $\epsilon$. The set of $\epsilon$-best replies is defined as:

$S^{\epsilon}(s)=\left\{s_{i} \in S_{i}\left(s_{-i}\right): u_{i}\left(s_{i} ; s_{-i}\right) \geq u_{i}\left(s_{i}^{\prime} ; s_{-i}\right)-\epsilon, \forall s_{i}^{\prime} \in S_{i}\left(s_{-i}\right)\right\}$.

An $\epsilon$-Nash equilibrium is a fixed point of $S^{\epsilon}$.

\subsection{The aggregated market and he $\epsilon$-best re- ply}

To find the utility-maximising bid combination for all auctions we need to introduce an alternative view of the market possibilities any bidder faces. Loosely speaking, an aggregated market "mimics" the behaviour of the individual auctions by defining a resource quantity and an allocation rule. The resource quantity is the sum of all resource quantities offered at all auctions. Our goal is to study the dynamics at each single auctions through our observation of an artificial market that would aggregate quantities as well as bids. In order to understand what the aggregated market is and how it functions, we introduce the utility-optimising $\epsilon$-best reply to such a market and prove its main properties. In a second step we propose a way in which a bidder can split his bid into the individual auctions.

To determine the utility-optimising $\epsilon$-best reply to the market a bidder $i$ has to consider his opponent bid profiles $s_{-i}^{(j)}$ from all auctions $J$. To merge all bid profiles into a common opponent market bid profile we propose the following procedure.

- For each auction $j$, create a vector called the opponent winning bid profile from auction $j$, with

$r_{-i}^{(j)}=\left[\left(a_{0}^{(j)}, p_{0}^{(j)}\right), . .,\left(a_{i-1}^{(j)}, p_{i-1}^{(j)}\right),\left(a_{i+1}^{(j)}, p_{i+1}^{(j)}\right), . .\left(a_{I}^{(j)}, p_{I}^{j}\right)\right]$.

The value for all $a_{i}^{(j)}$ 's can be derived with $a_{n}^{(j)}=$ $\left[Q^{(j)}-\sum_{k \neq n ; p_{k}^{(j)}>p_{n}^{(j)}}^{I} q_{k}^{(j)}\right]^{+}$, which is the PSP allocation rule. Since the bid representing the reserve price is defined as $\left(Q^{(j)}, p_{0}^{(j)}\right)$, we can safely assume that $\sum q_{i}^{(j)} \geq Q^{(j)} \forall j$. Therefore, $\sum_{i=0}^{I} a_{n}^{(j)}=Q^{(j)}$.

\footnotetext{
${ }^{3}$ We use the term iterative rather than dynamic since players do not devise a contingency plan or strategy over multiple rounds of the game but only react to the given opponent bid profile from the last round.
}

- Merge all vectors $r_{-i}^{(j)}$ into a common matrix $r_{-i}$, defined as as

$$
\begin{aligned}
& r_{-i}=\left(\begin{array}{c}
r_{-i}^{(1)} \\
. . \\
r_{-i}^{(J)}
\end{array}\right)=\left(\begin{array}{cc}
\left(a_{0}^{(1)}, p_{0}^{(1)}\right), & . .,\left(a_{i-1}^{(1)}, p_{i-1}^{(1)}\right), \\
. \cdot & \\
\left(a_{0}^{(J)}, p_{0}^{(J)}\right), & . .,\left(a_{i-1}^{(J)}, p_{i-1}^{(J)}\right),
\end{array}\right. \\
& \left.\begin{array}{ccc}
\left(a_{i+1}^{(1)}, p_{i+1}^{(1)}\right), & . ., & \left(a_{I}^{(1)}, p_{I}^{(1)}\right) \\
. . & \\
\left(a_{i+1}^{(J)}, p_{i+1}^{(J)}\right), & . ., & \left(a_{I}^{(J)}, p_{I}^{(J)}\right)
\end{array}\right) .
\end{aligned}
$$

In contrast to the matrix $s_{-i}$ the new matrix $r_{-i}$ contains all "winning" shares of the opponents' bids together with the unit-price if player $i$ were not present in the auction. Therefore, $r_{-i}$ can be seen as the opponent bid profile, which includes the capacity constraints in each market. By only including the winning shares a player is able to evaluate how much capacity in total can be obtained from all markets with his individual valuation for the sum of resources.

To gain a better understanding why we need to redefine $s_{-i}$ to create the "opponent market bid profile" a simple example is presented

EXAmPle 3.1. Consider a scenario with two auctions and total resources of $Q^{(1)}=Q^{(2)}=10$ and three bidders, all of whom have access to both auctions. Now, consider the situation of player 1, which demand function is given by $\theta^{\prime}(q)=10-q$. From each auction the player receives an opponents' bid profile $s_{-1}^{(1)}=[(9,11),(14,10)]$ and $s_{-1}^{(2)}=$ $[(8,2),(14,1)]$, respectively. To calculate his truthful reply to the market he needs to translate both opponent bid profiles into a common "opponent market bid profile". By just merging both opponent bid profiles he derives $\left(s_{-1}^{(1)}, s_{-1}^{(2)}\right)=s_{-1}=$ $[(9,11),(14,10),(8,2),(14,1)]$. With this opponent bid profile and the overall resources available in the market given by $Q=\sum_{j=1}^{2} Q^{(j)}=20$, he can derive his truthful reply to be $t_{1}=(0,10)$ (Figure 1). However, the merged market bid profile does not consider the constraints given by the distribution of resources between the two auctions. ${ }^{4}$ Since

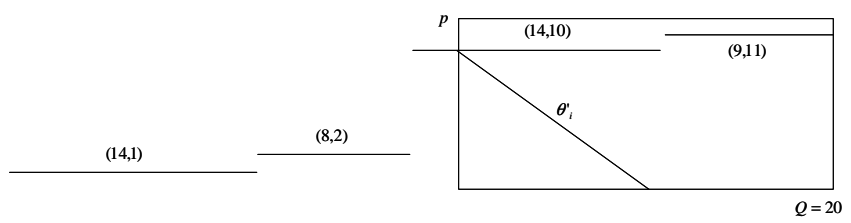

Figure 1: Graphical representation of $s_{-i}$.

in auction 1 unit-prices are very high but the total capacity of auction 1 is 10, and in contrast, unit-prices are very low in auction 2, player 1 will be able to acquire resources from the second market. Therefore, the opponent bid profiles need to be redefined in order to reflect the individual constraints in each auction. By using the above procedure we derive $r_{-1}=[(9,11),(1,10),(8,2),(2,1)]$. By using $r_{-1}$ for calculating a utility-maximising bid we derive $t_{1}=(8,2)$ (Figure

${ }^{4}$ The opponent bid profile $s_{-1}$ implies that the first two bids win a positive amount while the other two bids are loosing bids. This is not what we need since the allocation is constrained by the capacity in both auctions and not by the overall capacity constraint given by the $Q$. 


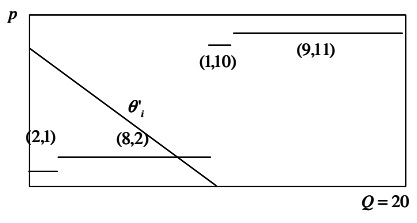

Figure 2: Graphical representation of $r$.

2). This bid reflects the player's truthful reply to the market because it considers the resource constraints from both auctions.

With the definition of $r_{-i}$ we can define the aggregated market.

DEFINITION 3.3. (The aggregated market)

An aggregated market can be defined by a resource $Q$ and an allocation rule $A$ with

$$
Q=\sum_{j=1}^{J} Q^{(j)}
$$

and

$$
A: \quad \begin{array}{rll}
S & \longrightarrow S \\
s=(q, p) & \longmapsto A(s)=(a(s), c(s)),
\end{array}
$$

with $S=\prod_{i \in I} S_{i}$. The allocation rule follows the PSP allocation rule of a single auction but uses the matrix $r_{-i}$ as the opponent bid profile. The $i$-th row of $A(s)$ is the allocation to player $i$, with $a_{i}(s)$ being the quantity and $c_{i}(s)$ being the overall cost.

$$
a_{i}\left(s_{i}, r_{-i}\right)=q_{i} \wedge \underline{Q}_{-i}\left(p_{i}, r_{-i}\right) \text {, }
$$

with $\underline{Q}_{-i}\left(y, r_{-i}\right)=\left[Q-\sum_{k=0, j=1, p_{k}>y}^{I, J} a_{k}^{(j)}\right]^{+}$.

$c_{i}(s)$ cannot be derived directly from $r_{-i}$ but only once the composite or split bids to the individual auctions have been defined.

In the following analysis we limit the strategy space to $T_{i}=\left\{s_{i} \in S_{i}: q_{i}=\sum^{(J)} q_{i}^{(j)} ; p_{i}=\theta_{i}^{\prime}\left(q_{i}\right)\right\}$ and search for a bid $t_{i} \in T_{i}$ as the truthful market reply.

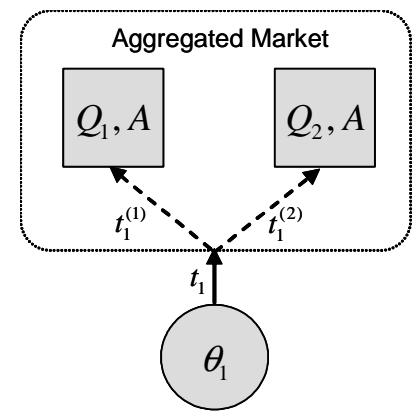

Figure 3: Graphical representation of the aggregated market bid $t_{i}$ and the bids $t_{i}^{(j)}$ to the single auctions.

We define truthful bidding in a way so that the sum of resource quantities a bidder expresses on all auctions at a given unit price corresponds to his demand function. While with this definition the truthful demand is not "visible" to a single auctioneer, the bidder ensures that he does not overbid in the aggregate market. Figure 3 expresses the difference between the aggregated market bid $t_{i}$ and the bids $t_{i}^{(j)}$ to the single auctions. The aggregated bid is a virtual construct and is only used internally by each bidder to derive the split bids to the auctions to ensure that the overall demand expressed in the market corresponds to the bidder's demand.

DeFinition 3.4. (Aggregated market bid)

Under assumption 3.1, a truthful reply to the market $t_{i}=$ $\left(v_{i}, w_{i}\right) \in T_{i} \cap S_{i}^{\epsilon}\left(s_{-i}\right)$ is given by

$$
v_{i}=\left[\sup G_{i}\left(r_{-i}\right)-\frac{\epsilon}{\theta_{i}^{\prime}(0)}\right]^{+} \text {and } w_{i}=\theta_{i}^{\prime}\left(v_{i}\right)
$$

with $\epsilon / \theta_{i}^{\prime}(0)$ being a factor by which each bid is reduced to avoid ties in bidding price between players and therefore, the utility from the aggregated market increases by at least $\epsilon,{ }^{5}$ where

$$
G_{i}\left(r_{-i}\right)=\left\{z \in\left[0, \sum_{j=1}^{J} Q^{(j)}\right]: z \leq Q_{i}\left(\theta_{i}^{\prime}(z), r_{-i}\right)\right\},
$$

with

$$
Q_{i}\left(y, r_{-i}\right)=\left[\sum_{j=1}^{J} Q^{(j)}-\sum_{p_{k}^{(j)}>y}^{I, J} a_{k}^{(j)}\right]^{+}
$$

Additionally, we define $\bar{v}_{i}=\left[\sup G_{i}\left(r_{-i}\right)\right]$ and $\bar{w}_{i}=\theta_{i}^{\prime}\left(v_{i}\right)$ to form $\bar{t}_{i}=\left(\bar{v}_{i}, \bar{w}_{i}\right)$.

Definition 3.4 is visualised in Figure 4.

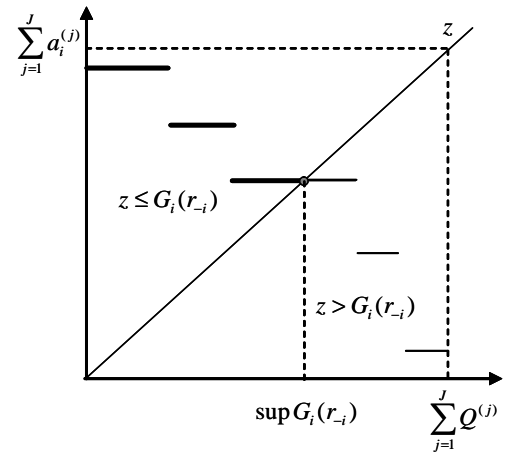

Figure 4: The graphical representation of $G_{i}\left(r_{-i}\right)$ and $\sup G_{i}\left(r_{-i}\right)$.

\subsection{Bid splitting and the BalancedBid strategy}

One key property of the single PSP auction is incentivecompatibility. We aim at understanding if bidders have incentives to reveal their true valuation in a market consisting of multiple PSP auctions. We therefore first present the bidding strategy, BalancedBid [5], which mimics truthful bidding when bidders are allowed to split their bids to bundle

${ }^{5}$ Note that this value has been interpreted as bid fee by [7]. However, in subsequent work [3], [4] it has been noted that this interpretation is potentially misleading. 
resources from several auctions. We then show that this bidding strategy is the myopic /epsilon-best response for all bidders in such a situation.

Once the truthful reply to the market has been determined, it is split into bids to be submitted to the individual auctions. We refer to such bids as BalancedBids. A balanced bid is defined as $t_{i}^{(j)}=\left(v_{i}^{(j)}, w_{i}\right)$, with $v_{i}^{(j)}$ being the quantity and $w_{i}$ being the unit price, bid at auction $j$, which has been derived from the (aggregated) market bid $t_{i}=\left(\sum^{J} v_{i}^{(j)}, w_{i}\right)$. The intuition behind the allocation rule at an auction $j$ is to sum all bids, received at $j$, which can be "beaten" by the unit price $\bar{w}_{i}$ determined by the market bid. ${ }^{6}$ Two cases have to be distinguished; in Case 1 the demand function $\theta_{i}^{\prime}$ does not intersect any of the bid steps. Case 2 describes the situation where the demand function crosses through one of the bid steps. Formally, we can state the two cases by defining

$$
\alpha_{i} \equiv \sum_{m=0, n=1 ; p_{n} \leq \bar{w}_{i}}^{I, J} a_{n}^{(m)}-\bar{v}_{i} .
$$

Now

$$
\begin{array}{ll}
\text { Case 1: } & \alpha_{i}=0 \\
\text { Case 2: } & \alpha_{i}>0 .
\end{array}
$$

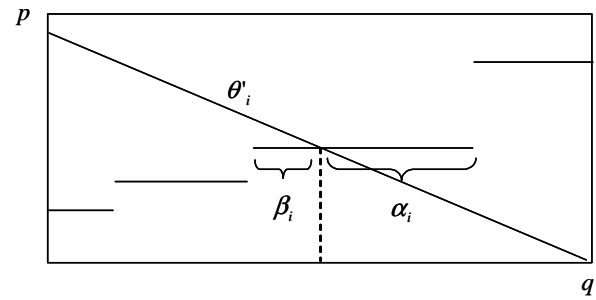

Figure 5: Visualisation of $\alpha_{i}$ and $\beta_{i}$.

We define $\beta_{i} \equiv \sum_{m=0, n=1 ; p_{n}=\bar{w}_{i}}^{I, J} a_{n}^{(m)}-\alpha_{i}$ as the share of the sum of all bids with unit price $\bar{w}_{i}$, which lies within the valuation of $i$ (see Figure 5). This segment can consist of multiple bids from different players and auctions. The following definition for deriving the single bids to the auctions is called BalancedBid

Definition 3.5. (BalancedBid)

[5] Under Assumption 3.1 and Definition 3.4 the balanced bid $t_{i}^{(j)}=\left(v_{i}^{(j)}, w_{i}\right)$ for player $i$ on auction $j$ is given by

$v_{i}^{(j)}=\left[\sum_{n=0, p_{n}^{(j)}<\bar{w}_{i}}^{I} a_{n}^{(j)}+\beta_{i} \frac{\sum_{n=0, p_{n}^{(j)}=\bar{w}_{i}}^{I} a_{n}^{(j)}}{\sum_{m=0, n=1 ; p_{n}^{(m)}=\bar{w}_{i}}^{I, J} a_{n}^{(m)}}-\frac{\epsilon}{J \theta_{i}^{\prime}(0)}\right]^{+}$ and

$$
w_{i}=\theta_{i}\left(\sum_{j=1}^{J} v_{i}^{(j)}\right)=v_{i} .
$$

${ }^{6}$ Note that we use $\bar{t}_{i}$ for this process as it gives us the truthful reply without the reduction by $\sum^{J} \epsilon / \theta_{i}^{\prime}(0)$. Otherwise, we would need to define a second constraint, which limits the balanced bids to the total quantity $v_{i}$.
Each balanced bid is reduced by $\epsilon / J \theta_{i}^{\prime}(0)$ to ensure that no ties between bidders can occur and that the utility derived from the sum of the bids from the $J$ auctions is increased by at least $\epsilon$.

One important question to ask is why a bidder should bid with identical unit prices on all auctions and not reduce the unit price to a level where he still wins the amount $q_{i}^{(j)}$. The reason for this can be found in the pricing rule of the PSP auction. Since a player is only charged with the cost of excluding other players from the market, the unit price does not influence the final charges. Since this unit price reflects the valuation of the total resources gained from the multi-auction market a player needs to use this price on all auctions.

We can now derive the cost of bid $t_{i}$, which consists of the sub-bids $t_{i}^{(j)}$ on each auction. Since we need to consider the resource constraints on each auction we have to calculate partial costs separately. For each auction, costs can be derived by calculating which demand has been excluded from receiving a positive allocation by the presence of bidder $i$. Formally, the costs are given by

$$
c_{i}\left(t_{i}, s_{-i}\right)=\sum_{j=0}^{J} \int_{0}^{a_{i}\left(t_{i}^{(j)}, s_{-i}^{(j)}\right)} P_{i}\left(z, s_{-i}^{(j)}\right) d z,
$$

where

$$
P_{i}\left(z, s_{-i}^{(j)}\right)=\inf \left\{y \geq 0: Q_{i}^{(j)}\left(y, s_{-i}\right) \geq z\right\}
$$

is the stair-case function $Q_{i}^{(j)}$ flipped by 90 degrees.

\subsection{Incentive-compatibility in a market con- sisting of multiple PSP auctions}

While it has been shown by [7] that the PSP auction is incentive-compatible the question remains if bidders still have an incentive for truthful bidding at the aggregated market. The splitting of a bid defined by BalancedBid seems to resemble truthful bidding in such a market. We need to show that for a myopic player, this strategy is the best strategy for a player compared to all other possible strategies to bid on multiple auctions.

Especially, we need to prove that there does not exist any other $s_{i} \in S_{i}\left(s_{-i}\right)$, which results in higher utility for player $i$. Since $s_{i}$ has been redefined to contain a vector of bids (to the different auction) instead of only one bid we need to define $a_{i}(s)=\sum^{J} a_{i}^{(j)}\left(s_{i}^{(j)} ; s_{-i}^{(j)}\right)$.

\section{Proposition 3.1. (Incentive compatibility)}

Under assumption 3.1, $\forall i \in I, \forall j \in J, \forall s_{-i}^{(j)} \in S_{-i}^{(j)}$, so that $Q_{i}\left(0, r_{-i}\right)=0$, for any $\epsilon>0$, the truthful reply $t_{i}$ defined in Definition 3.4 is an $\epsilon$-best reply to the market.

In the following we will show that for any $s_{i} \in S_{i}$ the yielded utility is equal or less to the utility gained from bidding $t_{i}$.

Proof. : (Incentive-compatibility)

$\forall s_{i} \in S_{i}\left(s_{-i}\right)$,

$$
\begin{aligned}
u_{i}\left(t_{i} ; s_{-i}\right)-u_{i}(s)= & \theta_{i}\left(a_{i}\left(t_{i} ; s_{-i}\right)\right)-c_{i}\left(t_{i} ; s_{-i}\right)- \\
& {\left[\theta_{i}\left(a_{i}(s)\right)-c_{i}(s)\right] } \\
= & \left.\int_{a_{i}\left(t_{i} ; s_{-i}\right)}^{a_{i}(s)}\left[P_{i}\left(z, r_{-i}\right)-\theta_{i}^{\prime}(z)\right] d \notin 2\right)
\end{aligned}
$$


Equation (2) is a consequence of the continuity of $\theta$. Note that the integral in (2) is always nonnegative because $\theta_{i}^{\prime}(q)$ is non-increasing in $q$ and $P_{i}(q)$ is non-decreasing in $q$. We can rewrite this property as $a_{i}\left(t_{i}, s_{-i}\right)=v_{i}$ and divide the integral in two parts by using $\bar{v}_{i}$.

$$
\begin{aligned}
= & \int_{\bar{v}_{i}}^{a_{i}(s)}\left[P_{i}\left(z, r_{-i}\right)-\theta_{i}^{\prime}(z)\right] d z+ \\
& \int_{v_{i}}^{\bar{v}_{i}}\left[P_{i}\left(z, r_{-i}\right)-\theta_{i}^{\prime}(z)\right] d z \\
\geq & \int_{\bar{v}_{i}}^{a_{i}(s)}\left[P_{i}\left(z, r_{-i}\right)-\theta_{i}^{\prime}(z)\right] d z-\epsilon .
\end{aligned}
$$

The inequality from (3) to (4) follows from the upper bound of $\left(\bar{v}_{i}-v_{i}\right) \leq \epsilon / \theta_{i}^{\prime}(0)$ and from the fact that $\theta_{i}^{\prime}$ is nonincreasing in $q$. Since $\epsilon$ is always positive we now need to show that bidding any value $v \notin\left[v_{i}, \bar{v}_{i}\right]$ and within the boundaries of $a_{i}(s)$ yields a utility $<\epsilon$ compared to the utility obtained by bidding $v=\bar{v}_{i}$. We graphically illustrate that this is the case.

Figure 6(a) depicts case 1 with $\bar{v}_{i}<a_{i}(s)$ and in Figure 6(b) the second case is shown when $\bar{v}_{i} \geq a_{i}(s)$. While in the first case the integrand is positive it is negative in the second case. But since the integral is calculated with switched boundaries $\left(\bar{v}_{i}>v\right)$, the integral turns to be positive.

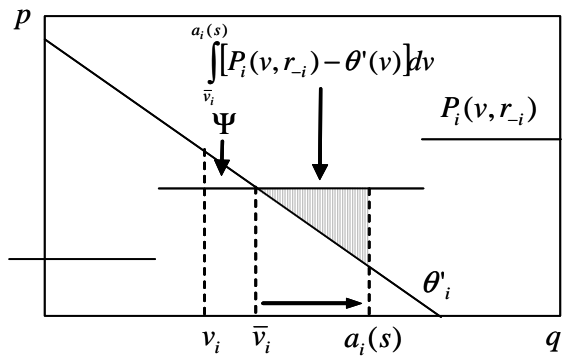

(a) Case 1: $\bar{v}_{i}<a_{i}(s)$

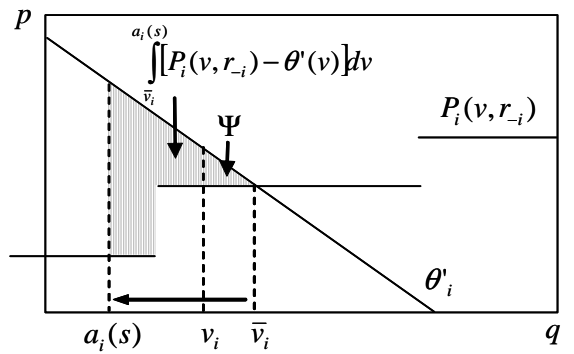

(b) Case 2: $\bar{v}_{i} \geq a_{i}(s)$

Figure 6: The graphical representation of the two cases for any value $z \notin\left[v_{i}, \bar{v}_{i}\right]$.

Case 1: $\left(\bar{v}_{i}<a_{i}(s)\right)$ : Take any $v \in\left(\bar{v}_{i}, a_{i}(s)\right]$. By definition of $v_{i}, v$ is not part of $G_{i}\left(r_{-i}\right)$. This leads to the conclusion that $c_{i}(s)=\int_{0}^{a_{i}(s)} P_{i}\left(\eta, r_{-i}\right) d \eta \geq \int_{0}^{v} P_{i}\left(\eta, r_{-i}\right) d \eta$. Therefore, $v$ must be larger than $Q_{i}\left(\theta_{i}^{\prime}(v)\right) . P_{i}\left(v, r_{-i}\right)$ has been defined as $P_{i}\left(v, r_{-i}\right)=\inf \left\{y \geq 0: Q_{i}\left(y, r_{-i}\right) \geq v\right\}$. Therefore, with a fixed opponent market bid profile $r_{-i}, \forall y$ and $v \geq 0$,

$$
v \leq Q_{i}\left(y, r_{-i}\right) \Rightarrow y \geq P_{i}\left(v, r_{-i}\right)
$$

and

$$
y>P_{i}\left(v, r_{-i}\right) \Rightarrow v \leq Q_{i}\left(y, r_{-i}\right)
$$

Because $v>Q_{i}\left(\theta_{i}^{\prime}(v)\right)$, Equation 6 implies that $\theta_{i}^{\prime}(v) \leq$ $P_{i}(v)$, which proves that integrand is $\geq 0$.

Case 2: $\left(\bar{v}_{i}>a_{i}(s)\right)$ : Take any $v \geq a_{i}(s)$. Because $\theta_{i}^{\prime}$ is non-increasing, $Q_{i}\left(., r_{-i}\right)$ is non-decreasing and $P_{i}\left(., r_{-i}\right) \geq$ 0 , any point to the left of $\bar{v}_{i}$ is in the set of $G_{i}\left(r_{-i}\right), \forall v<$ $v_{i}, v \in G_{i}\left(s_{-i}\right)$. Therefore, we have $v \leq Q_{i}\left(\theta_{i}^{\prime}(v), r_{-i}\right)$. By Equation (6) $\theta_{i}^{\prime}(v) \geq P_{i}\left(v, r_{-i}\right)$, which shows that the integrand in Equation (3) is $\leq 0$. But since the integral is calculated from right to the left, the integral becomes $\geq 0$.

\subsection{Continuity of the best reply to the market}

Another property of the best market reply, which is required to show that the proposed bidding strategy is part of a Nash equilibrium is the continuity of the best reply $t_{i}$ in opponent bid profiles $r_{-i}$. [7] has shown the continuity of the $\epsilon$-best reply in $s_{-i}$ for the one-auctioneer case. It is argued that for all $i \in I$, the $\epsilon$-best reply $t_{i}$ is continuous in $s_{-i}$ on any subset $V_{i}(\bar{P}, \underline{P})=\left\{s_{-i} \in S_{-i}: \forall z>0, \bar{P} \geq\right.$ $\left.P_{i}\left(z, s_{-i}\right) \geq \underline{P}\right\}$, with $\infty \geq \bar{P} \geq \underline{P}>0$. It remains to proof this property for the proposed truthful bidding strategy on the set of opponent market profiles $R_{-i}$.

Before we can proceed we need to prove that $R_{-i}$ is a compact subset of $S_{-i}$. To analyse the properties of $r_{-i}$ we define the mapping $h$, which maps $s_{-i}$ to $r_{-i}$.

$$
\begin{aligned}
h: & \left(S_{-i}\right)^{J} \longrightarrow\left(S_{-i}\right)^{J} \\
& s_{-i} \longmapsto h\left(s_{-i}\right)=r_{-i}
\end{aligned}
$$

and define a set $R_{-i}$ as

$$
R_{-i}=\left\{r_{-i} \mid r_{-i}=h\left(s_{-i}\right) \text { for some } s_{-i}^{(j)} \in S_{-i}\right\} .
$$

Lemma 3.1. $R_{-i}$ is a compact subset of $S_{-i}$, and is therefore bounded and closed.

Proof. In a first step we only consider the first component $a_{n}^{(j)}$ of the mapping $h$, i.e.

$\left(q_{0}^{(j)}, \ldots, q_{I}^{(j)}\right) \mapsto a_{n}^{(j)}\left(q_{0}^{(j)}, \ldots, q_{I}^{(j)}\right):=\left[Q^{(j)}-\sum_{k \neq n ; p_{k}^{(j)}>p_{n}^{(j)}}^{I} q_{k}^{(j)}\right]^{+}$.

This map is continuous as the concatenation of continuous maps. We define

$A_{-i}:=\left\{a_{n}^{(j)}\left(q_{0}^{(j)}, \ldots, q_{I}^{(j)}\right) \mid\left(q_{0}^{(j)}, \ldots, q_{I}^{(j)}\right) \in\left(\left[0, \max _{j} Q^{(j)}\right]\right)^{I}\right\}$.

Since $A_{-i}$ is the continuous image of the compact set $\left(\left[0, \max _{j} Q^{(j)}\right]\right)^{I}$ it is compact itself. Now the set $R_{-i}$ is given by

$$
R_{-i}=\left(A_{-i} \times[0, \bar{P}]\right)^{I \times J} .
$$

Thus it is compact as the cartesian product of compact sets.

Proposition 3.2. (Continuity of the best reply to the aggregated market)

The truthful bid to the aggregated market $t_{i}$ is continuous in $r_{-i}$ on a subset $V_{i}(\underline{P}, \bar{P})=\left\{r_{-i} \in R_{-i}: \forall v>0, \bar{P} \geq\right.$ $\left.P_{i}\left(v, r_{-i}\right) \geq \underline{P}\right\}$, with $\infty>\bar{P} \geq \underline{P}>0$. 
Proof. Since we have shown in Lemma 3.1 that $R_{-i}$ is a compact subset of $S_{-i}, t_{i}$ must be continuous in $r_{-i}$. We refer to the detailed proof in [7] for the continuity of $t_{i}$ in $s_{-i}$.

The continuity of the best market reply does not necessarily mean that the bids to the single auctions are continuous in the opponent bid profiles $s_{-i}^{(j)}$. While we have first experienced the discontinuity during simulation experiments (see e.g., [6]) with the BalancedBid strategy, we have also been able to understand this analytically. Two main practical reasons can be identified. First, we consider the "symmetrical" case in which all players have access to all auctions. In this setting the reason for discontinuity in bids to single auctions lies in the asynchronism of information when forming the best reply to the market. If an opponent bid profile $s_{-i}^{(j)}$ arrives with a delay, a player may use bid profiles from different time periods to form the new reply. Therefore, he may switch his demand to one of the auctions with the more attractive opponents bids.

The second reason for a large change in single bids can occur in a situation when not all players have access to all markets. In this case opponent bid profiles differ in the number of elements and players and can force a player to switch his demand rapidly from one auction to the other with only a small change in one opponent bid profile. The following example illustrates this case.

EXAmple 3.2. (Discontinuity in the reply to the single auctions)

Consider two auctions with $Q=10$ and 3 players, all having access to both auctions. We now analyse the best reply of player 1 and the bid profiles $s^{(1)}=s^{(2)}=([8,2],[6,4],[5,6])$ and $s^{(1)}=([8,2],[5.9,4.1],[5,6])$. The resulting opponent market bid profile is $r_{-1}=([5,6],[5,6],[5,4],[5,4])$ and $r_{-1}^{\prime}=$ $([5,6],[5,6],[5,4.1],[5,4])$. If we assume a demand function $\theta_{1}^{\prime}=10-q$ for player 1 , we can derive the best reply to the market, resulting in $t_{1}=(5.9,4.1)$ and $t_{1}^{\prime}=(5.8,4.2){ }^{7}$ We can see that a small change in one of the bid profiles does let the best reply to the market change only marginally. However, if we calculate the bids to the auctions we derive $t_{1}^{(1)}=(2.95,4.1), t_{1}^{(2)}=(2.95,4.1)$ and $t_{1}^{\prime(1)}=(5,4.2), t_{1}^{\prime(2)}=$ $(0.8,4.2)$, resulting in a large change in how the demand is distributed between auctions. Since now the first auction becomes more attractive to player 1, he shifts his demand to this market.

\subsection{Nash equilibrium of the game}

With Definition 3.2 we have already established the notion of Nash equilibrium in a general sense. We could also show that a market consisting of multiple PSP auctions is still incentive-compatible, namely incentivises players to reveal their true valuation to the aggregated market. The remaining question is if the equilibrium of the iterative game is of Nash type.

To restrict our attention to truthful bidding to the market as defined in (3.4) we set a reserve price $p_{0}>0$. This implies that for all $i \in I, Q_{i}\left(y, r_{-i}\right)=0$, for all $y<p_{0}$. Then, Proposition 3.1 is fulfilled and allows us to restrict our attention to truthful bidding, which are still best replies to the aggregate market. As described by [7], this forms

\footnotetext{
${ }^{7}$ Assuming some $\epsilon$
}

an embedded game within the larger game, with the strategy space being $T \subset S$, the feasible set for player $i$ being $T_{i} \cap S_{i}\left(s_{-i}\right)$, and the best replies $X_{i}^{\epsilon}(s)=T_{i} \cap S_{i}^{\epsilon}(s)$. If we can find a fixed point of $X^{\epsilon}$ in $T$, this must also be a fixed point of $S^{\epsilon}$ in $S$.

Proposition 3.3. (Nash equilibrium of the iterative game) In the auction game consisting of multiple, independent PSP auctions, a reserve price $p_{0}>0$, and players finding their best reply to the aggregated market according to Definition 3.4 and Definition 3.5, if Assumption 3.1 holds, then for any $\epsilon>0$, there exists a truthful $\epsilon$-Nash equilibrium $s^{*} \in T$.

Proof. We now provide a sketch of the proof, which closely follows the work by [7].

We have shown the continuity of the truthful reply $t_{i}=$ $\left(v_{i}, w_{i}\right)$ to the aggregate market in $r_{-i}$ on $R_{-i}$ in Proposition 3.2. Because $\theta_{i}^{\prime}$ is continuous (by Assumption 3.1), $v_{i}\left(q_{i}, p_{i}\right)=v_{i}\left(q_{i}, \theta_{i}^{\prime}\left(q_{i}\right)\right)$ can be viewed as a continuous mapping of $\left[0, \sum Q^{(j)}\right]^{I}$ onto itself (for reference, see [7]). It can now be shown by Brouwer's fixed point theorem that any continuous mapping of a convex compact set into itself has at least one fixed point $\left(\forall i, \exists q_{i}^{*}=v_{i}\left(q_{i}^{*}\right) \in\left[0, \sum Q^{(j)}\right]^{I}\right)$. Therefore, an equilibrium $s^{*}=t\left(s^{*}\right) \in T$ must exist.

In [3] it is proved that the difference between the market clearing price and the maximum price bid by any bidder at a given round can be arbitrarily bounded. The bound is a linear function of the square root of $\epsilon$. If $\epsilon$ is sufficiently small, PSP provides an approximation to the market clearing price. In other words, prices set by bids at a $\epsilon$-Nash equilibrium are arbitrarily close to prices at Nash-equilibrium. The latter implies that the aggregated measure of welfare at a $\epsilon$-Nash equilibrium is also arbitrarily close to the aggregated measure of welfare at Nash equilibrium. Because the Nash equilibrium achieved by PSP is efficient, the iterative bidding process is arbitrarly close to the welfare of the efficient equilibrium.

\subsection{Properties of the individual PSP auctions in aggregate equilibrium}

After having shown the properties of the market consisting of multiple PSP auctions we can now proceed in better understanding the implications of the BalancedBid strategy (Definition 3.5) to form the bids to the auctions. One property we have already explored is the discontinuity of $t_{i}^{(j)}$ in $S_{-i}^{(j)}$. This means that small changes in the opponent bid profile can lead to large changes in the balanced to the auctions. Additionally, in equilibrium, it may be possible to shift demand between auctions without disturbing the aggregate equilibrium. We show this by a simple example.

EXAMPLE 3.3. In this example we define two auctions with $Q^{(1)}=15, Q^{(2)}=5$ and two bidders, both having access to both auctions. The utility functions of both bidders are $\theta_{1}^{\prime}=-0.05 q+1$ and $\theta_{1}^{\prime}=-0.1 q+1$, respectively. With $\epsilon$ being small we can derive a possible $\epsilon$-Nash equilibrium of the aggregated market with the bids $(13.4,0.33)$ for bidder 1 and $(6.8,0.32)$ for bidder 2 , resulting in the allocation $(13.4,0.064)$ and $(6.6,0)$, assuming that bidder 1 has last updated his bid and that bidder 2 cannot further improving his surplus by updating his bid. 
With the equilibrium solution for the aggregate market being identified, a consequent problem is the definition of the balanced bids for both bidders. The BalancedBid strategy (as defined in Definition 3.5) distributes the demand between auctions proportionally to the bids equal in unit price, which cross the marginal demand of a bidder. ${ }^{8}$ However, other possible combinations of demand allocations lead to the same result if the aggregate market is in equilibrium.
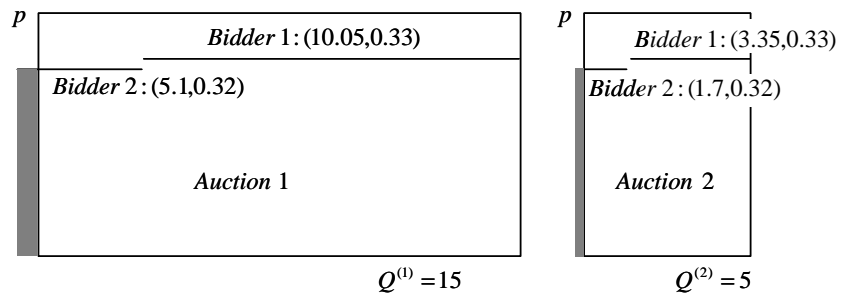

(a) Case 1: Demand is equally distributed between auctions, as defined in Definition 3.5.

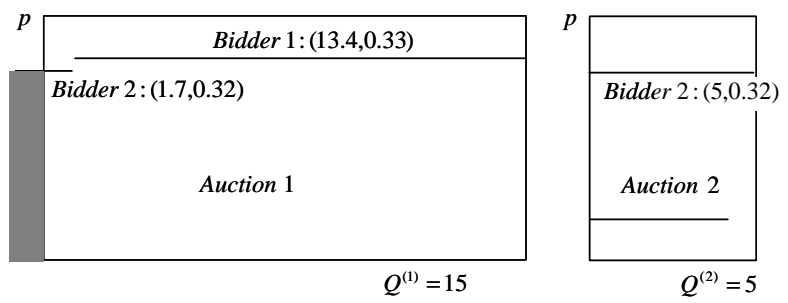

(b) Case 2: Demand is shifted so that bidder 1 receives resources from auction 1 and bidder 2 receives resources from auction 2 .

Figure 7: Two different allocations of demand between auctions belonging to the same equilibrium solution for the aggregated market.

Figure 7 depicts two possible combinations of demand allocations between the two auctions, leading to the same equilibrium result for the aggregated market as defined before. The first case shows how Definition 3.5 allocates the demand between the auctions (assuming zero delay in the distribution of opponent bid profiles), resulting in balanced bids defines as $(10.05,0.33),(3.35,0.33)$, and $(5.1,0.32),(1.7,0.32)$, respectively. Case 2 shows an alternative allocation in which bidder 1 shifts his demand to auction 1, while bidder 2 receives the remaining resources from auction 1 and the full resources from auction $2((13.4,0.33),(0,0.33)$, and $(1.8,0.32),(5,0.32))$. Both combinations result in the same social welfare (total social welfare and individual surplus) and summed revenue for the auctioneers. However, in the first case, auctioneer 1 receives all revenues while in case 2, the revenue is distributed over both auctioneers.

It needs to be mentioned that shift in revenue from both providers to only one provider, as shown in the example, is irrelevant in the sense that PSP serves as congestionavoidance mechanism and is not intended to generate significant revenues in equilibrium. By choosing a sufficiently small $\epsilon$ the revenue in equilibrium will be small as users reduce their demand in order to avoid any congestion. Instead,

${ }^{8}$ With only two bidders in the market there exist only two opponent bids coming from the same bidder, which therefore always cross the marginal demand function of the bidder. by setting an appropriate reserve price, a provider is able to define a fixed charge for each unit of allocated resources in equilibrium.

The above example shows that it is possible to shift the demand of bidders between auctions without changing the fundamental results of the aggregated equilibrium. This also illustrates the difficulty in defining the notion of equilibrium in each single auction if users' preferences are expressed by a one-dimensional demand function. While we can speak of a stable situation on all single auctions, due to the possibility of allocation shifting, it is not possible to derive a notion of equilibrium.

While with the proposed bidding strategy we could obtain an efficient allocation of resources in aggregate, the possibility of exchanging allocation shares between auctions can be further examined in understanding possible post-market situations. For example, if providers can express a preference for certain allocation patterns, such as a preference for holding the minimum number of active users in a system, a wholesale situation may arise, in which providers swap user allocations to improve their utility in another dimension.

\section{CONCLUSION}

We have presented a bidding strategy in a market consisting of multiple second-price auctions for network resources in form of a divisible good, when bidders have elastic demand and are indifferent of combination they receive from each auction. The bidding strategy resembles truthful bidding in a setting with multiple, simultaneous auctions and allows bidders to balance their demand between such auctions. While the bids to the individual auctions do not reveal the truthful overall demand of a bidder, the aggregated bid has the properties of a truthful reply; the aggregated bid expresses a bidder's marginal valuation of a bidder for the aggregate of resources. We could show that, under some assumptions about the properties of a bidders utility function, the BalancedBid bidding strategy maximises a bidder's utility. A bidder cannot do any better without risking to overbid in at least one auction. We could show that if all bidders employ this bidding strategy, the aggregated market converges to an efficient Nash equilibrium.

The proposed bidding strategy applies to all situations in which multiple, non-cooperative sellers offer divisible goods, such as bandwidth, on an open market, which is accessible for all bidders. While the bidding strategy has been developed with the focus on providing a solution for dynamic resource allocation in wireless networks, the model can also be transfered to other fields such as distributed computing.

A possible future extension of this research is to introduce a preference relations for the combination of resources a bidder receives. For example, a bidder may express a preference for a larger quantity of resources from a single auction compared to smaller shares from several auctions. With this extension resources become less fragmented between bidders and the convergence period may be shortened.

\section{REFERENCES}

[1] R. Berezdivin, R. Breinig, and R. Topp.

Next-generation wireless communications concepts and technologies. IEEE Communications Magazine, 40(3):108-16, 2002.

[2] V. Krishna. Auction theory. Academic Press, 2004. 
[3] P. Maillé. Market clearing price and equilibria of the progressive second price mechanism. Technical Report 1522, IRISA, 2003.

[4] P. Maillé and B. Tuffin. Multi-bid auctions for bandwidth allocation in communication networks. In Proceedings of the 23rd IEEE Infocom Conference, Hong-Kong, China, March 2004.

[5] M. Roggendorf and F. Beltran. Flow-based resource allocation in a multiple-access wireless market using an auction. In Proceedings of the Second International Workshop on Incentive-Based Computing (IBC), July 4-7, Lisbon, Portugal, 2006.

[6] M. Roggendorf, F. Beltran, and J. Gutierrez. Architecture and implementation of an agent-based simulation tool for market-based pricing in next-generation wireless networks. In Proceedings of the TridentCOM 2006, 2nd International Conference on Testbeds and Research Infrastructures for the Development of Networks and Communities, March 1-3, Barcelona, Spain, pages 282 - 288, 2006.

[7] N. Semret. Market mechanisms for network resource sharing. PhD thesis, Columbia University, Center for Telecommunications Research, 1999.

[8] B. Tuffin. Revisited progressive second price auctions for charging telecommunication networks. Telecommunication Systems, 20(3-4), 2002.

[9] D. Zeng, J. Cox, and M. Dror. Coordination of purchasing and bidding activities across markets. In Proceedings of the 37th Hawaii International Conference on SystemSciences, 2004. 